\title{
XỬ LÝ ĐỐI TƯợNG THỬA ĐẤT CÓ CẠNH LÀ ĐƯờNG CONG
}

\author{
ThS. ĐINH HẢI NAM, ThS. PHAM THÉ HUYNH, TS.TRÀN THÙY DƯO'NG \\ Trường Đại học Mỏ - Địa chất
}

\section{Tóm tắt:}

Đối tượng đường cong là một đối tượng mới được đưa vào quản lý ranh giới thưa đất. Khi đưa đối tượng này vào quản lý phát sinh nhiều vấn đề cần giải quyết trong công tác thành lập bản đồ số địa chính. Bài báo này đưa ra giải pháp xử lý một số vấn đề liên quan đến việc quản lý thửa đất có cạnh là đường cong. Đây là tiền đề để xây dựng chương trình đồ họa độc lập khi quan tâm đến yếu tố đường cong trên cạnh thửa đất.

\section{Mở đầu}

Theo các quy định kỹ thuật trước đây về công tác thành lập bản đồ địa chính, sơ đồ thửa đất được thể hiện bằng các đoạn thẳng, các đoạn cong đều được lấy gần đúng là các đoạn thẳng. Với cách lấy gần đúng này sơ đồ thửa đất sẽ có quá nhiều đỉnh thửa trên chỗ đoạn cong làm cho khó thể hiện chính xác kích thước các cạnh. Đến nay theo thông tư 23/2014/TT-BTNMT "Quy định về Giấy chứng nhận quyền sử dụng đất, quyền sở hữu nhà ở và tài sản khác gắn liền với đất" [4] cho phép thể hiện sơ đồ thửa đất có cạnh là đường cong, chính vì vậy vấn đề nghiên cứu xử lý thửa đất có cạnh là đường cong hết sức cần thiết.

\section{Giải quyết vấn đề}

\subsection{Giải pháp xử lý thửa đất có cạnh là đường cong}

Đối tượng đường cong là một đối tượng rất phức tạp với nhiều hình dạng khác nhau, chính vì vậy, giải pháp xử lý thửa đất có cạnh là đường cong là biến đường cong thành các đối tượng đơn giản hơn nhưng vẫn đảm bảo yếu tố cong của nó. Giải pháp được đưa ra như sau: đường cong được phân tách thành một hoặc nhiều cung tròn nối tiếp nhau tiệm cận nhất đối với đường cong. Do đó, vấn đề trọng tâm cần nghiên cứu là đối tượng cung tròn.

\section{2. Đối tượng cung tròn}

\subsubsection{Các yếu tố cơ bản của cung tròn}

Một cung tròn nối hai điểm $\mathrm{P}_{1}$ và $\mathrm{P}_{2}$ với quy ước thống nhất đi theo ngược chiều kim đồng hồ từ $\mathrm{P}_{1}$ đến $\mathrm{P}_{2}$ (hình 1 ). (Xem hình 1 )

- Điểm tâm cung tròn: $P_{C}\left(X_{c}, Y_{c}\right)$

- Bán kính: $R$

- Điểm đầu: $P_{1}\left(X_{1}, Y_{1}\right)$

- Điểm cuối: $P_{2}\left(X_{2}, Y_{2}\right)$

- Góc bắt đầu: $A_{1}$ là góc nghiêng của đoạn thẳng nối tâm và điểm đầu

Người phản biện: TS. Dương Chí Công 


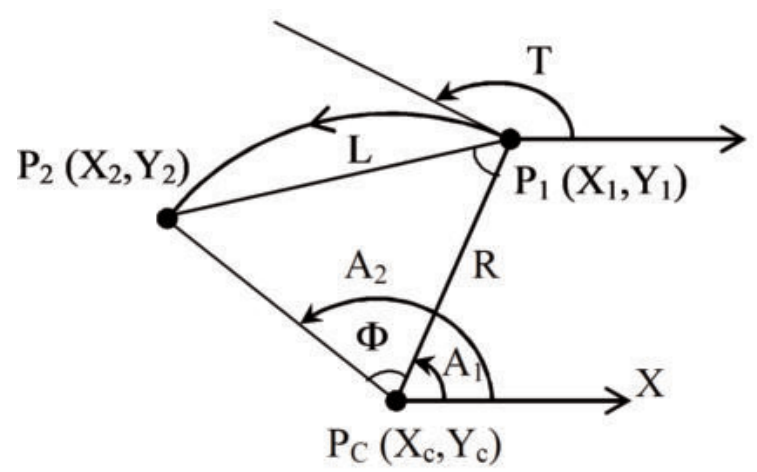

Hình 1: Các yếu tố cơ bản của cung tròn

- Góc kết thúc: $A_{2}$ là góc nghiêng của đoạn thẳng nối tâm và điểm cuối

- Góc chắn cung: $\mathrm{F}=\mathrm{A}_{2}-\mathrm{A}_{1}$

- Chiều dài dây cung: L

- Góc nghiêng của hướng tiếp tuyến tại điểm đầu: T

Góc nghiêng được hiểu là góc hợp bởi trục hoành và đoạn thẳng với chiều dương ngược chiều kim đồng hồ.

\subsubsection{Quản lý cung tròn}

Một cung tròn có các yếu tố nêu trên, tuy nhiên để dựng một cung tròn có thể có nhiều cách dựng khác nhau sao cho xác định duy nhất một cung tròn. Cách quản lý là xác định tổ hợp cơ bản các yếu tố: tọa độ của điểm tâm, điểm đầu, điểm cuối. Các tổ hợp khác được tính toán đưa về tổ hợp cơ bản bằng các phép biến đổi hình học.

2.2.3. Biến đổi tổ hợp các yếu tố cung tròn về tổ hợp cơ bản

(a) Tổ hợp ba điểm không thẳng hàng

Giả sử cung tròn đi qua 3 điểm $P_{1}\left(X_{1}, Y_{1}\right), P_{2}\left(X_{2}, Y_{2}\right), P_{3}\left(X_{3}, Y_{3}\right)$ không thẳng hàng với $P_{1}$ là điểm đầu, $\mathrm{P}_{3}$ là điểm cuối, $\mathrm{P}_{2}$ là điểm nằm trên cung tròn. Cần xác định tọa độ tâm $\left(\mathrm{X}_{\mathrm{c}}\right.$, $\left.Y_{c}\right)$.

Theo [2] xác định tọa độ $\left(X_{c}, Y_{c}\right)$ của tâm đường tròn ngoại tiếp một tam giác như sau:

$$
\begin{aligned}
& X_{C}=\frac{\left(X_{1}^{2}+Y_{1}^{2}\right)\left(Y_{3}-Y_{2}\right)+\left(X_{2}^{2}+Y_{2}^{2}\right)\left(Y_{1}-Y_{3}\right)+\left(X_{3}^{2}+Y_{3}^{2}\right)\left(Y_{2}-Y_{1}\right)}{2\left(\mathbf{Y}_{1}\left(X_{2}-X_{3}\right)+\mathbf{Y}_{2}\left(X_{3}-X_{1}\right)+\mathbf{Y}_{3}\left(X_{1}-X_{2}\right)\right)} \\
& Y_{C}=\frac{\left(X_{1}^{2}+Y_{1}^{2}\right)\left(X_{2}-X_{3}\right)+\left(X_{2}^{2}+Y_{2}^{2}\right)\left(X_{3}-X_{1}\right)+\left(X_{3}^{2}+Y_{3}^{2}\right)\left(X_{1}-X_{2}\right)}{2\left(\mathbf{Y}_{1}\left(X_{2}-X_{3}\right)+\mathbf{Y}_{2}\left(X_{3}-X_{1}\right)+\mathbf{Y}_{3}\left(X_{1}-X_{2}\right)\right)}
\end{aligned}
$$

(b). Tổ hợp điểm đầu, tâm, góc chắn cung

Khi biết các yếu tố điểm đầu $P_{1}\left(X_{1}, Y_{1}\right)$, tâm $P_{C}\left(X_{c}, Y_{c}\right)$ và góc chắn cung $F$. Cần xác điểm cuối $\mathrm{P}_{2}\left(\mathrm{X}_{2}, \mathrm{Y}_{2}\right)$. 


$$
\begin{aligned}
& \text { Ta có } \mathrm{R}=\sqrt{\left(X_{c}-X_{1}\right)^{2}+\left(Y_{c}-Y_{1}\right)^{2}} \\
& \mathrm{~A}_{1}=\arctan \left(\left(\mathrm{Y}_{1}-\mathrm{Y}_{\mathrm{C}}\right) /\left(\mathrm{X}_{1}-\mathrm{X}_{\mathrm{C}}\right)\right. \\
& \mathrm{A}_{2}=\mathrm{A}_{1}+\mathrm{Q} \\
& \text { tính được } \quad \mathrm{X}_{2}=\mathrm{X}_{\mathrm{c}}+\mathrm{R}{ }^{*} \operatorname{Cos}\left(\mathrm{A}_{2}\right) \\
& \mathrm{Y}_{2}=\mathrm{Y}_{\mathrm{C}}+\mathrm{R} * \operatorname{Sin}\left(\mathrm{A}_{2}\right)
\end{aligned}
$$

(c). Tổ hợp điểm đầu, tâm, chiều dài dây cung

Khi biết các yếu tố điểm đầu $P_{1}\left(X_{1}, Y_{1}\right)$, tâm $P_{C}\left(X_{c}, Y_{C}\right)$ và chiều dài dây cung $L$. Cần xác điểm cuối $\mathrm{P}_{2}\left(\mathrm{X}_{2}, \mathrm{Y}_{2}\right)$.

Xác định được $\mathrm{R}$ theo công thức (3)

Từ đó xác định được góc chắn cung $\mathrm{F}$ theo công thức:

$$
F=\arccos \left(1-L^{2} /\left(2 R^{2}\right)\right)
$$

Tọa độ điểm $P_{2}$ được xác định theo các công thức: (4), (5), (6), (7).

(d). Tổ hợp điểm đầu, điểm cuối, góc chắn cung

Khi biết các yếu tố điểm đầu $P_{1}\left(X_{1}, Y_{1}\right)$, điểm cuối $P_{2}\left(X_{2}, Y_{2}\right)$ và góc chắn cung $F$. Cần xác định tâm $\mathrm{P}_{\mathrm{C}}\left(\mathrm{X}_{\mathrm{c}}, \mathrm{Y}_{\mathrm{c}}\right)$.

Xác định chiều dài dây cung $L$ theo công thức:

$$
\mathrm{L}=\sqrt{\left(X_{2}-X_{1}\right)^{2}+\left(Y_{2}-Y_{1}\right)^{2}}
$$

Từ (8) suy ra $R=\frac{L}{\sqrt{2(1-\operatorname{Cos}(\Phi))}}$

Gọi $\beta$ là góc hợp bởi dây cung và bán kính (hình 1).

Ta có: $\quad \beta=\frac{\pi-\phi}{2}$

Gọi $A_{3}$ là góc nghiêng của đoạn $P_{1} P_{2}$

$$
A_{3}=\arctan \left(\left(Y_{2}-Y_{1}\right) /\left(X_{2}-X_{1}\right)\right.
$$

tính được $X_{C}=X_{1}+R{ }^{*} \operatorname{Cos}\left(\beta+A_{3}\right)$

$$
Y_{C}=Y_{1}+R * \operatorname{Sin}\left(\beta+A_{3}\right)
$$

(e). Tổ hợp điểm đầu, điểm cuối, góc nghiêng của hướng tiếp tuyến tại điểm đầu

Khi biết các yếu tố điểm đầu $P_{1}\left(X_{1}, Y_{1}\right)$, điểm cuối $P_{2}\left(X_{2}, Y_{2}\right)$ và góc nghiêng của hướng tiếp tuyến tại điểm đầu $T$. Cần xác định tâm $P_{C}\left(X_{c}, Y_{c}\right)$.

Gọi $\beta$ là góc hợp bởi dây cung và bán kính.

Gọi $A_{3}$ là góc nghiêng của đoạn $P_{1} P_{2}$ được tính theo công thức (11) 
Ta có: $\beta+A_{3}=\frac{\pi}{2}+T$

$$
\beta=\frac{\pi}{2}+T-A_{3}
$$

Góc chắn cung $\mathrm{Q}=\pi-2^{*} \beta$

Bán kính $\mathrm{R}$ tính theo công thức (9) và (10)

$$
\text { tính được } \begin{aligned}
X_{C} & =X_{1}+R * \operatorname{Cos}\left(\frac{\pi}{2}+T\right) \\
Y_{C} & =Y_{1}+R * \operatorname{Sin}\left(\frac{\pi}{2}+T\right)
\end{aligned}
$$

(f). Tổ hợp điểm đầu, điểm cuối, bán kính

Khi biết các yếu tố điểm đầu $P_{1}\left(X_{1}, Y_{1}\right)$, điểm cuối $P_{2}\left(X_{2}, Y_{2}\right)$ và bán kính $R$. Cần xác định tâm $\mathrm{P}_{\mathrm{C}}\left(\mathrm{X}_{\mathrm{c}}, \mathrm{Y}_{\mathrm{c}}\right)$.

Xác định chiều dài dây cung $L$ theo công thức (9)

Từ đó xác định được góc chắn cung $\Phi$ theo công thức (8)

Tổ hợp này trở thành tổ hợp (d).

\subsubsection{Các cách dựng hình cung tròn}

Sử dụng tổ hợp cơ bản (điểm đầu, điểm cuối, tâm) để dựng hình các cung tròn. Với các phân tích ở trên có thể dựng hình bằng các tổ hợp (a), (b), (c), (d), (e), (f). Ngoài ra, có thể dựng được cung tròn nối tiếp đoạn thẳng hoặc cung tròn.

- Cung tròn nối tiếp đoạn thẳng: điểm đầu $\mathrm{P}_{1}$ của cung tròn cần dựng là điểm cuối của đoạn thẳng, hướng tiếp tuyến tại điểm đầu $T$ là góc nghiêng của đoạn nối điểm cuối và điểm đầu của đoạn thẳng. Khi đó chỉ cần chỉ ra điểm cuối của cung tròn và dựng hình theo tổ hợp (e).

- Cung tròn nối tiếp cung tròn: điểm đầu $\mathrm{P}_{1}$ của cung tròn cần dựng là điểm cuối của cung tròn trước đó, hướng tiếp tuyến tại điểm đầu $T$ là hướng tiếp tuyến của cung tròn trước đó. Khi đó chỉ cần chỉ ra điểm cuối của cung tròn và dựng hình theo tổ hợp (e).

\subsubsection{Xác định điểm thuộc cung tròn}

Một cung tròn $C$ xác định bởi điểm đầu $P_{1}\left(X_{1}, Y_{1}\right)$, điểm cuối $P_{2}\left(X_{2}, Y_{2}\right)$ và tâm $P_{C}\left(X_{c}\right.$, $\left.Y_{c}\right)$.

Xác định điểm $\mathrm{P}_{\mathrm{i}}\left(\mathrm{X}_{\mathrm{i}}, \mathrm{Y}_{\mathrm{i}}\right)$ có thuộc cung tròn này không?

Tính bán kính cung tròn $\mathrm{R}$ theo công thức (3)

Tính khoảng cách $d$ từ $P_{i}$ đến $P_{C}$ theo công thức:

$$
\mathrm{d}=\sqrt{\left(X_{i}-X_{c}\right)^{2}+\left(Y_{i}-Y_{c}\right)^{2}}
$$

+ Nếu $\mathrm{d}<\mathrm{R}$ thì $\mathrm{P}_{\mathrm{i}}$ nằm phía trong đường tròn chứa cung tròn $\mathrm{C}$

+ Nếu $\mathrm{d}>\mathrm{R}$ thì $\mathrm{P}_{\mathrm{i}}$ nằm phía ngoài đường tròn chứa cung tròn $\mathrm{C}$ 


\section{Nghiên cúu}

+ Nếu $\mathrm{d}=\mathrm{R}$ thì $\mathrm{P}_{\mathrm{i}}$ nằm trên đường tròn chứa cung tròn $\mathrm{C}$

Tính góc nghiêng $A_{1}$ theo công thức (4).

Tính góc nghiêng $A_{2}$ theo công thức:

$$
A_{2}=\arctan \left(\left(Y_{2}-Y_{c}\right) /\left(X_{2}-X_{c}\right)\right)
$$

Tính góc nghiêng $A_{i}$ theo công thức:

$$
A_{i}=\arctan \left(\left(Y_{i}-Y_{c}\right) /\left(X_{i}-X_{c}\right)\right)
$$

Để đảm bảo $A_{2}$ luôn lớn hơn $A_{1}$ thì khi $A_{2}$ nhỏ hơn $A_{1}$ ta cộng thêm một lượng $2 * \pi$ vào $A_{2}$.

- Nếu $A_{1} \leq A_{i} \leq A_{2}$ thì $P_{i}$ thuộc cung tròn $C$

- Nếu không thì $\mathrm{P}_{\mathrm{i}}$ không thuộc cung tròn $\mathrm{C}$

\subsubsection{Xác định hình chữ nhật nhỏ nhất chứa cung tròn}

Việc xác định được hình chữ nhật bao quanh một cung tròn giúp ta giải quyết một số bài toán trong biểu diễn đồ họa máy tính như thu toàn bộ bản vẽ vừa cửa sổ hiển thị, thể hiện sơ đồ thửa đất có cung tròn trên một khổ giấy. Để xác định một hình chữ nhật nhỏ nhất chứa một đối tượng đa giác thì rất đơn giản vì các cạnh của đa giác gồm tọa độ điểm đầu và điểm cuối. Hình chữ nhật cần xác định bởi hai điểm có tọa độ $\left(X_{\max }, Y_{\max }\right)$ và $\left(X_{\min }\right.$, $Y_{\min }$ ) là hoành độ và tung độ lớn nhất của các điểm đầu, điểm cuối các đoạn thẳng.

Phương pháp xác định tọa độ hình chữ nhật nhỏ nhất $\mathrm{H}$ chứa cung tròn như sau:

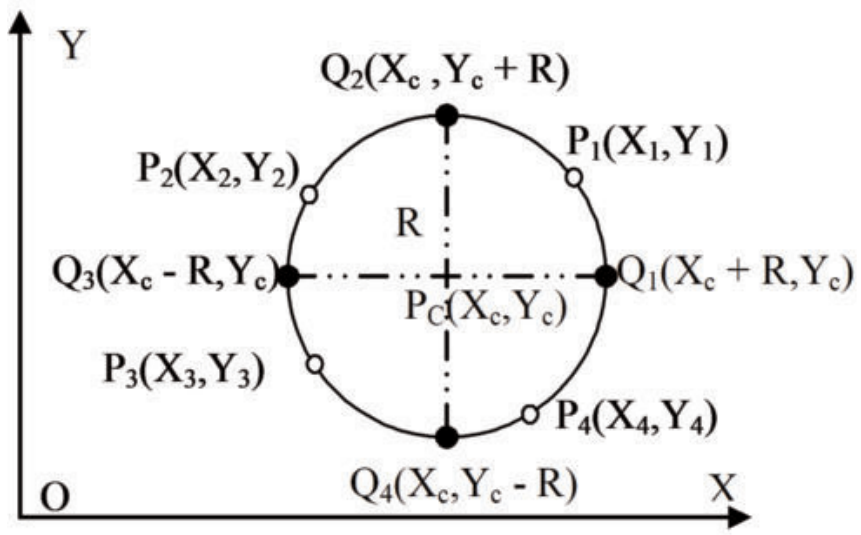

Hình 2: Các điểm đặc biệt của đường tròn

Một cung tròn được xác định bởi điểm đầu $\mathrm{P}_{1}\left(\mathrm{X}_{1}, \mathrm{Y}_{1}\right)$, điểm cuối $\mathrm{P}_{2}\left(\mathrm{X}_{2}, \mathrm{Y}_{2}\right)$ và tâm $\mathrm{P}_{\mathrm{C}}\left(\mathrm{X}_{\mathrm{C}}, \mathrm{Y}_{\mathrm{C}}\right)$.

* Xác định $\mathrm{R}$ theo công thức (3).

* Xác định tọa độ các điểm một phần tư đường tròn $Q_{1}, Q_{2}, Q_{3}, Q_{4}$ như hình 2. 


\section{Nghiên cúu}

* Kiểm tra giữa điểm đầu và điểm cuối của cung tròn có chứa các điểm $Q_{1}, Q_{2}, Q_{3}, Q_{4}$ nào không theo mục (2.2.5). Từ đó, xác định được các điểm $Q_{1}, Q_{2}, Q_{3}, Q_{4}$ có thuộc cung tròn nối $P_{1}$ và $P_{2}$ không.

- Nếu không chứa điểm một phần tư nào thì hình chữ nhật $\mathrm{H}\left(\mathrm{X}_{\max }, \mathrm{Y}_{\max }\right)$ và $\left(\mathrm{X}_{\min }, \mathrm{Y}_{\min }\right)$ xác định như sau:

$$
\begin{aligned}
& X_{\text {max }}=\max \left(X_{1}, X_{2}\right) ; Y_{\max }=\max \left(Y_{1}, Y_{2}\right) \\
& X_{\text {min }}=\min \left(X_{1}, X_{2}\right) ; Y_{\text {min }}=\min \left(Y_{1}, Y_{2}\right)
\end{aligned}
$$

(với hàm $\max ($ ) là hàm xác định giá trị lớn nhất, hàm min() là hàm tìm giá trị nhỏ nhất)

- Nếu chứa các điểm một phần tư nào thì đưa các điểm đó vào để so sánh với $\mathrm{P}_{1}$ và $\mathrm{P}_{2}$ tìm $\left(X_{\max }, Y_{\max }\right)$ và $\left(X_{\min }, Y_{\min }\right)$.

\subsubsection{Kiểm tra giao của đoạn thẳng và cung tròn}

Muốn kiểm tra giao của đoạn thẳng nối 2 điểm $\mathrm{P}_{\mathrm{m}}\left(\mathrm{X}_{\mathrm{m}}, \mathrm{Y}_{\mathrm{m}}\right)$ và $\mathrm{P}_{\mathrm{n}}\left(\mathrm{X}_{\mathrm{n}}, \mathrm{Y}_{\mathrm{n}}\right)$ với cung tròn trước hết ta kiểm tra giao của đoạn thẳng với đường tròn $O$ chứa cung tròn $C$ tâm $\mathrm{P}_{\mathrm{C}}\left(\mathrm{X}_{\mathrm{c}}, \mathrm{Y}_{\mathrm{c}}\right)$.

Xác định $\mathrm{P}_{\mathrm{m}}$ và $\mathrm{P}_{\mathrm{n}}$ xem nằm trong hay nằm ngoài đường tròn $\mathrm{O}$ (phân tích trong mục 2.2.5).

Xác định khoảng cách từ tâm của $\mathrm{O}$ đến đoạn thẳng $\mathrm{P}_{\mathrm{m}} \mathrm{P}_{\mathrm{n}}$ theo công thức:

$$
D=\frac{\left|\left(Y_{m}-Y_{n}\right) *\left(X_{c}-X_{m}\right)+\left(X_{m}-X_{n}\right) *\left(Y_{c}-Y_{m}\right)\right|}{\sqrt{\left(Y_{m}-Y_{n}\right)^{2}+\left(X_{m}-X_{n}\right)^{2}}}
$$

Xác định $\mathrm{R}$ theo (3)

+ Nếu $\mathrm{D}>\mathrm{R}$ thì đoạn thẳng $\mathrm{P}_{\mathrm{m}} \mathrm{P}_{\mathrm{n}}$ không cắt đường tròn $\mathrm{O}$;

+ Nếu $\mathrm{D}=\mathrm{R}$ thì đoạn thẳng $\mathrm{P}_{\mathrm{m}} \mathrm{P}_{\mathrm{n}}$ tiếp xúc với đường tròn $\mathrm{O}$;

+ Nếu $D<R$ thì xét tiếp các trường hợp:

- Nếu $\mathrm{P}_{\mathrm{m}}, \mathrm{P}_{\mathrm{n}}$ cùng nằm trong đường tròn $\mathrm{O}$ thì đoạn thẳng $\mathrm{P}_{\mathrm{m}} \mathrm{P}_{\mathrm{n}}$ không cắt đường tròn $\mathrm{O}$

- Nếu $\mathrm{P}_{\mathrm{m}}, \mathrm{P}_{\mathrm{n}}$ một điểm nằm trong đường tròn, một điểm nằm ngoài đường tròn $\mathrm{O}$ thì đoạn thẳng $\mathrm{P}_{\mathrm{m}} \mathrm{P}_{\mathrm{n}}$ cắt đường tròn $\mathrm{O}$;

- Nếu $P_{m}, P_{n}$ cùng nằm ngoài đường tròn $O$ thì cần so sánh khoảng cách $D_{m n}$ giữa $P_{m}$ và $P_{n}$ với khoảng cách $D_{t}$ từ $P_{m}$ tới tiếp điểm kẻ từ $P_{m}$ đến $O$. Nếu $D_{m n}<D_{t}$ thì đoạn thẳng $\mathrm{P}_{\mathrm{m}} \mathrm{P}_{\mathrm{n}}$ không cắt đường tròn $\mathrm{O}$; Nếu $\mathrm{D}_{\mathrm{mn}} \geq \mathrm{D}_{\mathrm{t}}$ thì đoạn thẳng $\mathrm{P}_{\mathrm{m}} \mathrm{P}_{\mathrm{n}}$ cắt đường tròn $\mathrm{O}$;

Như vậy, xác định được đoạn thẳng $\mathrm{P}_{\mathrm{m}} \mathrm{P}_{\mathrm{n}}$ có cắt đường tròn $\mathrm{O}$ hay không.

Nếu $\mathrm{P}_{\mathrm{m}} \mathrm{P}_{\mathrm{n}}$ không cắt đường tròn $\mathrm{O}$ thì tất nhiên $\mathrm{P}_{\mathrm{m}} \mathrm{P}_{\mathrm{n}}$ cũng không cắt cung tròn $\mathrm{C}$; 
Nếu $P_{m} P_{n}$ cắt đường tròn $O$ thì cần xác định giao điểm $P_{m} P_{n}$ với $O$. Nếu ít nhất một giao điểm này thuộc $\mathrm{C}$ (mục 2.2.5) thì đoạn thẳng $\mathrm{P}_{\mathrm{m}} \mathrm{P}_{\mathrm{n}}$ cắt $\mathrm{C}$, nếu không có giao điểm nào thuộc $\mathrm{C}$ thì đoạn thẳng $\mathrm{P}_{\mathrm{m}} \mathrm{P}_{\mathrm{n}}$ không cắt $\mathrm{C}$.

\subsubsection{Xác định điểm nằm trong thửa đất có chứa cung tròn}

Điểm $\mathrm{P}$ cần xác định có tọa độ $\left(\mathrm{X}_{\mathrm{P}}, \mathrm{Y}_{\mathrm{P}}\right)$

Gọi điểm $P_{\max }$ có tọa độ $\left(X_{\max }+1000, Y_{P}\right)$ với $X_{\max }$ là hoành độ lớn nhất của hình chữ nhật bao tất cả các thửa đất. Xác định số lượng các giao điểm $\mathbf{n}$ của đoạn thẳng $\mathrm{PP}_{\max }$ với các cạnh thửa đất. Với các cạnh thửa đất là cung tròn thì áp dụng phân tích ở mục 2.2.7 để xác định giao.

- Nếu $\mathbf{n}$ là số lẻ thì điểm xét sẽ nằm trong thửa đất;

- Còn nếu $\mathbf{n}$ là số chẵn thì điểm xét nằm ngoài thửa đất.

\subsubsection{Tính diện tích thửa đất có chứa cung tròn}

a). Tính diện tích hình thang khi có cung tròn.

Hình thang 1-d-2-2'-1' chứa cung tròn $d$ đi qua điểm 2 và điểm 1 . Cung tròn này có tâm là $\mathrm{P}_{\mathrm{C}}$, điểm đầu là điểm 2 , điểm cuối là điểm 1 .

Để tính diện tích hình thang này cần xác định diện tích hình viên phân 1-d-2 đánh dấu trên hình 3.

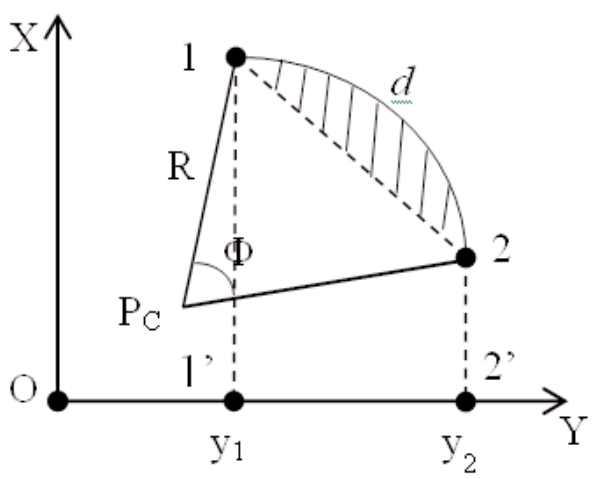

Hình 3: Hình thang chứa cung tròn

Tính bán kính cung tròn $\mathrm{R}$ theo công thức (3); góc chắn cung $\$$ theo công thức (9), (8).

$$
\begin{aligned}
& S_{1-d-2-2^{\prime}-1^{\prime}}=S_{1-2-2^{\prime}-1^{\prime}}+S \\
& S_{1-2-2^{\prime}-1^{\prime}}=\left(x_{1}+x_{2}\right)\left(y_{2}-y_{1}\right)
\end{aligned}
$$

Diện tích hình viên phân $S_{1-d-2}$ được tính bằng diện tích hình quạt 1-d-2- $P_{C}$ trừ đi diện tích tam giác 1-2- $P_{C}$ và bằng $\frac{1}{2} R^{2}(\Phi-\sin \Phi)[3]$

- Nếu hướng tính diện tích ngược chiều với chiều của cung tròn thì $S=\frac{1}{2} R^{2}(\Phi-\sin \Phi)$

- Nếu hướng tính diện tích cùng chiều với chiều của cung tròn thì $S=-\frac{1}{2} R^{2}(\Phi-\sin \Phi)$

b). Tính diện tích thửa đất có cạnh là cung tròn 
Trên hình 4 là một ví dụ một thửa đất có chứa cung tròn ở cạnh 1-2; hướng tính diện tích theo chiều 1-d-2-3-4-1 cùng chiều kim đồng hồ.

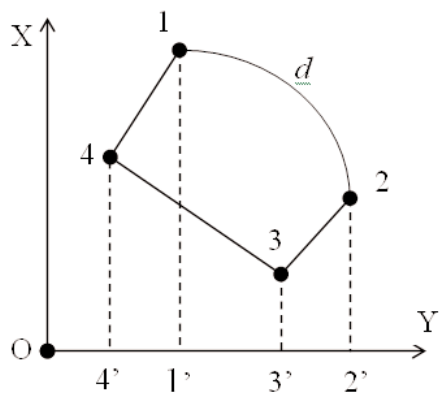

Hình 4: Thửa đất chứa cung tròn

Diện tích thửa đất được tính như sau:

$$
\mathrm{S}_{1-\mathrm{d}-2-3-4}=\mathrm{S}_{1-\mathrm{d}-2-2^{\prime}-1^{\prime}}+\mathrm{S}_{2-3-3^{\prime}-2^{\prime}}+\mathrm{S}_{3-4-4^{\prime}-3^{\prime}}+\mathrm{S}_{4-1-1^{\prime}-4^{\prime}}
$$

Các đại lượng $S_{2-3-3^{\prime}-2}$, $S_{3-4-4 '-3}$, $S_{4-1-1 '-4}$ tính diện tích theo công thức (21); còn $S_{1-d-2-2^{\prime}-1}$ ' tính theo công thức (20) với $S=\frac{1}{2} R^{2}(\Phi-\sin \Phi)$

\section{Kết luận}

Qua các phân tích trong bài báo cho thấy, đối tượng đường cong khi đưa vào quản lý ranh giới thửa đất hết sức phức tạp. Tuy nhiên, với các giải pháp, phương pháp tính và xác định các yếu tố đã trình bày là tiền đề để xây dựng chương trình đồ họa độc lập của Việt Nam khi quan tâm đến yếu tố đường cong trên cạnh thửa đất với các chức năng như dựng hình, hiển thị bản vẽ, tính diện tích, chọn vùng... $\bigcirc$

\section{Tài liệu tham khảo}

[1]. Võ Văn Thành, Đàm Văn Chương (1997), Hướng dẫn lập trình Windows API với Visual Basic. Tập 1,2,3, NXB Thống kê.

[2]. Vera B. Anand (2000), Đồ họa máy tính và mô hình hóa hình học, NXB TP Hồ Chí Minh.

[3]. http://vi.wikipedia.org/wiki/Cung_\%28h\%C3\%ACnh_h\%E1\%BB\%8Dc\%29

[4]. Bộ Tài nguyên và Môi trường (2014), thông tư 23/2014/TT-BTNMT "Quy định về Giấy chứng nhận quyền sử dụng đất, quyền sở hữu nhà ở và tài sản khác gắn liền với đất", trang 44, Hà Nội.O

\section{Summary}

\section{Parcel object processing with curve edges}

MSc. Dinh Hai Nam, MSc. Pham The Huynh, Dr. Tran Thuy Duong

Hanoi University of Mining and Geology

Curve is new object used for presenting parcel boundary edge. When use this object, there are a lot of problems need to be solved in cadastral mapping. This paper presents a solution to solve some problems concerning with curve edges of parcel. This is a basic to build a graphic application when using the curve for presenting the edges of parcel. $\bigcirc$

Ngày nhận bài: 03/9/2014. 